\title{
Bone Marrow Mesenchymal Stem Cell Administration Significantly Improves Outcome after Retinal Ischemia in Rats
}

\author{
Biji Mathew, Ph.D. ${ }^{1}$, Jacqueline N. Poston, M.D. ${ }^{2}$, John C. Dreixler, Ph.D. ${ }^{2}$, Leianne Torres, \\ M.D. ${ }^{1}$, Jasmine Lopez, B.S. ${ }^{1}$, Ruth Zelkha, B.S. ${ }^{3}$, Irina Balyasnikova, Ph.D. ${ }^{4}$, Maciej S. \\ Lesniak, M.D. ${ }^{4}$, and Steven Roth, M.D. ${ }^{1,2}$ \\ ${ }^{1}$ Department of Anesthesiology, University of Illinois at Chicago \\ ${ }^{3}$ Department of Ophthalmology, University of Illinois at Chicago \\ ${ }^{2}$ Department of Anesthesia and Critical Care, University of Chicago \\ ${ }^{4}$ Department of Neurosurgery, Northwestern University, Chicago, IL
}

\begin{abstract}
Purpose-Ischemia-associated retinal degeneration is one of the leading causes of vision loss and to date, there are no effective treatment options. We hypothesized that delayed injection of bone marrow stem cells (BMSCs) $24 \mathrm{~h}$ after the onset of ischemia could effectively rescue ischemic retina from its consequences, including apoptosis, inflammation, and increased vascular permeability, thereby preventing retinal cell loss.

Methods-Retinal ischemia was induced in adult Wistar rats by increasing intraocular pressure (IOP) to $130-135 \mathrm{~mm} \mathrm{Hg}$ for $55 \mathrm{~min}$. BMSCs harvested from rat femur were injected into the vitreous $24 \mathrm{~h}$ post-ischemia. Functional recovery was assessed 7 days later using electroretinography (ERG) measurements of the a-wave, b-wave, P2, scotopic threshold response (STR), and oscillatory potentials (OP). The retinal injury and anti-ischemic effects of BMSCs were quantitated by measuring apoptosis, autophagy, inflammatory markers, and retinal-blood barrier permeability. The distribution and fate of BMSC were qualitatively examined using realtime fundus imaging, and retinal flat mounts.
\end{abstract}

Results-Intravitreal delivery of BMSCs significantly improved recovery of the ERG a- and bwaves, OP, negative STR, and P2, and attenuated apoptosis as evidenced by decreased TUNEL and caspase-3 protein levels. BMSCs significantly increased autophagy, decreased inflammatory

\footnotetext{
Address correspondence to: Steven Roth, M.D., Department of Anesthesiology, MC 515, University of Illinois Medical Center, 1740 West Taylor Street, Chicago, Illinois 60612, rothgas@uic.edu, 312-996-0052 (telephone), 773-753-1024 (fax).

Conflict of Interest:

All authors certify that they have no affiliations with or involvement in any organization or entity with any financial interest (such as honoraria; educational grants; participation in speakers' bureaus; membership, employment, consultancies, stock ownership, or other equity interest; and expert testimony or patent-licensing arrangements), or non-financial interest (such as personal or professional relationships, affiliations, knowledge or beliefs) in the subject matter or materials discussed in this manuscript.

Ethical approval: Animal Experiments:

All applicable international, national, and/or institutional guidelines for the care and use of animals were followed. All procedures performed in studies involving animals were in accordance with the ethical standards of the Animal Care Committees of the Universities of Chicago and Illinois.
} 
mediators (TNF-a, IL-1 $\beta$, IL-6), and diminished retinal vascular permeability. BMSCs persisted in the vitreous and were also found within ischemic retina.

Conclusions-Taken together, our results indicate that intravitreal injection of BMSCs rescued the retina from ischemic damage in a rat model. The mechanisms include suppression of apoptosis, attenuation of inflammation and vascular permeability, and preservation of autophagy.

\section{Keywords}

apoptosis; autophagy; bone marrow mesenchymal stem cells; electroretinography; inflammation; ischemia; retinal vascular permeability

\section{Introduction}

Retinal ischemic injury from diseases such as diabetic retinopathy and retinal artery occlusion, with irreversible damage to retinal cells, is one of the major causes of vision loss. Ischemia is associated with generation of reactive oxygen species [1], upregulation of inflammatory cytokines and chemokines [2], and infiltration of inflammatory cells [3], as well as retinal vascular leakage [4]. Few efficacious treatment modalities are available to reverse the damage or to prevent loss of retinal cells and improve function [5]. However, emerging studies focusing on adult stem cell therapy, using harvested BMSCs, a heterogeneous stem cell mixture, offer promising new directions to treat retinal damage [6]. Stem cell therapy has been limited by cell survival, migration and implantation into the retina, as well as limited understanding of their mechanisms of action. Beside their capacity for self-renewal and differentiation into neuronal cells, transplanted BMSCs release paracrine factors for enhanced endogenous neuroprotection and neuro-repair, which may be the principal mechanisms of action [7]. Mesenchymal stem cells exhibit increased adherence to vascular endothelium in response to chemokines, adhesion molecules, and matrix metalloproteases and home to injury sites, rendering them ideal for treatment of retinal ischemic disorders [8].

Previously, we reported the protective effect of BMSC hypoxic conditioned medium in our rat model of retinal ischemic injury and demonstrated the neuroprotective effect of media in augmenting retinal function and preventing retinal cell loss. Post ischemic prosurvival signaling triggered by the media may play an important role in neuroprotection [9]. While BMSC media is promising, a limitation is that there is no accompanying source for ongoing release of the secretome (i.e., the cells). Therefore, injection of BMSCs, once optimal timing, safety, and mechanisms of action are determined, may be an alternate method for neuroprotection. Accordingly, $\mathrm{Li}$ et al demonstrated improved histological recovery with mouse and rat bone marrow stem cells injected shortly after the induction of ischemia [10]. However, delayed injection would be a more typical scenario in patients with acute retinal vascular occlusion, while stem cell treatment for diabetic retinopathy (DR) would most likely have to be initiated before the onset of irreversible neuronal and vascular endothelial damage. Direct administration into vitreous remains the optimal delivery method because of the limited number of cells reaching the retina after intravenous injection as well as the risks of embolic phenomena. 
Based on our recent work applying ischemic post-conditioning or injection of BMSC conditioned media $24 \mathrm{~h}$ after ischemia, we hypothesized that delayed application of BMSCs, $24 \mathrm{~h}$ after ischemia, could significantly improve recovery outcomes [9]. Towards this aim, and using autologous BMSCs, we evaluated the underlying mechanisms responsible for the protective effects of BMSCs in retinal ischemia, in our previously characterized rat model. This ischemia model is directly relevant to acute retinal ischemia as from retinal vascular occlusion, but it also recapitulates many of the features of diabetic retinopathy [11].

\section{Methods}

\subsection{Electroretinography}

These and all other procedures conformed to the Association for Research in Vision and Ophthalmology Resolution on the Use of Animals in Research and were approved by the Institutional Animal Care and Use Committees of the University of Chicago and the University of Illinois. Dark adaptation was achieved by placing rats in complete darkness for at least $2 \mathrm{~h}$ before electroretinogram (ERG) recording; previous work by others have shown it is adequate to dark adapt the retina [12]. For baseline and post-ischemic (i.e., after 7 days) follow-up ERG, rats were injected i.p. with ketamine ( $35 \mathrm{mg} / \mathrm{kg})$, and xylazine $(5 \mathrm{mg} / \mathrm{kg})$ every $20 \mathrm{~min}$. Corneal analgesia was with 2 drops of $0.5 \%$ proparacaine. Pupils were dilated with one drop of $0.5 \%$ tropicamide (Alcon, Fort Worth, TX), and cyclomydril (Cyclopentolate hydrochloride $0.2 \%$, with phenylephrine hydrochloride $1 \%$, Alcon). Further details on our methods have been reported previously [13-15].

The ERG was recorded from both eyes of each animal at baseline (prior to the experiments) and at 7 days after ischemia. This timing was followed in order to be consistent with our previous studies where the post-ischemic follow up was at 7 days; we followed procedures that we previously described $[9,16] . \mathrm{Ag} / \mathrm{AgCl}$ electrodes were fashioned from 0.010 " Teflon-coated silver wire (Grass Technologies, West Warwick, RI). Approximately $10 \mathrm{~mm}$ was exposed and fashioned into a small loop to form the corneal/positive electrodes while $\sim 20 \mathrm{~mm}$ of wire was exposed to form a hairpin loop, the sclera/negative electrodes looped around the eye. Electrodes were first attached to a $9 \mathrm{~V}$ battery and placed in a $1 \mathrm{~N} \mathrm{HCl}$ bath for $12 \mathrm{~s}$ until coated with $\mathrm{AgCl}$. Electrodes were referenced to a $12 \mathrm{~mm} \times 30$ gauge stainless steel, needle electrode (Grass) inserted 2/3 down the length of the rat tail [17]. Electrode impedance was unchanged during recordings. Stimulus-intensity ERG recordings were obtained using a UTAS-E 4000 ERG system and a Ganzfeld (LKC Technologies, Gaithersburg, MD) [16].

The low pass filter was $0.05 \mathrm{~Hz}$ and high $500 \mathrm{~Hz}$. For the scotopic threshold response (STR), which was performed first, flash series intensities varied electronically, and using neutral density filters secured into frames with edges sealed with black tape to prevent light leakage and signal contamination, from $-6.4 \mathrm{~cd} \cdot \mathrm{s} \cdot \mathrm{m}^{-2}$ to $-5.42 \mathrm{log} \mathrm{cd} \cdot \mathrm{s} \cdot \mathrm{m} .{ }^{-2}$ Responses were averaged from 60 flashes $1 \mathrm{~s}$ apart [18]. For the stimulus-intensity a- and b-waves, flash intensities were $-3.39 \mathrm{log} \mathrm{cd} \cdot \mathrm{s} \cdot \mathrm{m}^{-2}$ to $1.40 \mathrm{log} \mathrm{cd} \cdot \mathrm{s} \cdot \mathrm{m}^{-2}$. We averaged 3-10 flashes delivered 4-27 s apart. The number of flashes decreased and time between them increased with intensity as we have previously reported [16]. Light intensity settings were confirmed by photometry (EG \& G Model 550 photometer, Electro-Optics, Boulder, CO, USA). 


\subsection{Retinal ischemia}

Male Wistar rats (200-250 gm, Harlan, Indianapolis, IN) were maintained on a $12 \mathrm{~h}$ on/12 h off light cycle. The eyes were treated with one drop each of topical Vigamox $(0.5 \%)$ and cyclomydril (see above), and for local anesthesia for needle placement, 2 drops of $0.5 \%$ proparacaine. For retinal ischemia, rats were anesthetized with chloral hydrate, $275 \mathrm{mg} / \mathrm{kg}$ i.p. After sterile preparation, and working under an operating microscope, a 30-gauge, 5/8inch metal needle (BD Precision Glide; Becton-Dickinson, Franklin Lakes, NJ) was placed with its tip directed away from the lens, just inside the anterior chamber of the right eye, which served as the experimental eye, while the left eye of the same rat was control eye. To enable simultaneous pressurization for increasing intraocular pressure (IOP), and measurement of IOP, the needle was connected by a length of plastic tubing via a three-way stopcock to an electronic pressure transducer (Transpac 42661-04-27; Abbott, North Chicago, IL) and to an elevated bag of balanced salt solution (BSS; by sterile technique, BSS was transferred from its bottle [Alcon] to an empty 1000-mL $0.9 \%$ saline plastic bag [Baxter, Deerfield, IL, USA]). IOP, continually displayed on a monitor (Hewlett-Packard HP78534C; Palo Alto, CA), was increased to $130-135 \mathrm{~mm} \mathrm{Hg}$ and maintained constantly for 55 minutes by pressurizing the bag (Smiths Medical Clear-Cuff, Dublin, OH) [9]. The temperature was maintained at $36-37^{\circ} \mathrm{C}$ using a servo-controlled heating blanket (Harvard Apparatus, Natick, MA). Oxygen saturation was measured by pulse oximetry (Ohmeda; Louisville, CO) with a Band-Aid type probe on the tail. Supplemental oxygen, when necessary to maintain $\mathrm{O}_{2}$ saturation $>93 \%$, was administered using a cannula in front of the nares and mouth.

\subsection{In vivo administration of BMSCs}

Isolation, propagation, and characterization of BMSCs were described previously [16]. Briefly, BMSCs in early passages were cultured in complete medium (MEM - alpha and HAMs-F12 (1:1), with 10\% fetal bovine serum) to 60-70\% confluency, then cells collected, washed with phosphate-buffered saline (PBS), and re-suspended in PBS right before the vitreal injection. With cells suspended in PBS, it was appropriate to use PBS for vehicle (control) experiments. Cells were counted and checked for viability using Trypan Blue. To minimize variability, the cells were derived from a single donor rat. The whole process of collecting the cells after culture, and injecting them to the vitreous was done in $45 \mathrm{~min}$ time.

For the injections, rats were anesthetized with i.p. ketamine $(35 \mathrm{mg} / \mathrm{kg})$ and xylazine $(5 \mathrm{mg} /$ $\mathrm{kg})$. BMSCs were injected into the mid-vitreous under microscopic guidance over 1 minute with a Hamilton syringe (Reno, NV) and a 3/8" 32-gauge (Becton-Dickinson) needle (4 $\mu \mathrm{l}$ cell suspensions in PBS, with $\sim 50,000$ cells $/ 4 \mu \mathrm{l}$ ) into both ischemic (right eye) and control (left eye) eyes $24 \mathrm{~h}$ post-ischemia. Sham control rats were administered PBS in both ischemic and non-ischemic eyes. Previous studies indicated no adverse effects, e.g., increased IOP or retinal detachment, from injections $[9,16]$.

The experimental protocol consisted of ERG baseline recordings, then, one eye was subjected to retinal ischemia. At $24 \mathrm{~h}$ after ischemia ended, both eyes (the ischemic eye and the non-ischemic paired control eye) were injected either with BMSCs (experimental group), 
while in the control group, the vehicle (PBS) was injected. The ERG recordings were repeated 7 days after ischemia.

\subsection{In vivo imaging of GFP-BMSCs}

Migration from the vitreous and implantation in the retina are challenges in stem cell therapy in the eye. To track the fate of BMSCs, we used rat green fluorescent-labeled BMSCs (GFP-BMSCs, Cyagen, Santa Clara, CA). The cells were cultured in complete medium (MEM - alpha and HAMs-F12 (1:1), with 10\% fetal bovine serum) to reach 60 $70 \%$ confluency. GFP-BMSCs were collected and re-suspended in PBS, and injected into the vitreous in $4 \mu \mathrm{l}$ PBS (with $\sim 50,000$ cells/ $4 \mu \mathrm{l}$ ). For in vivo real-time imaging, rats were injected i.p. with ketamine ( $35 \mathrm{mg} / \mathrm{kg})$, and xylazine $(5 \mathrm{mg} / \mathrm{kg}$ ). Pupils were dilated as described in previous sections, above. Fluorescent fundus images were obtained with a Micron IV Retinal Imaging Microscope (Phoenix Research Labs, Pleasanton, CA), 1, 7, and 21 days' post injection of GFP-BMSCs.

\subsection{Fluorescent imaging and localization of GFP-labeled BMSCs in retinal flat mounts}

GFP-BMSC injected ischemic and normal eyes were enucleated, the retina was separated from the eyecup and flat mounted on a gelatin coated slide. Retinal flat-mounts were washed several times in PBS, DAPI was applied to the surface for $5 \mathrm{~min}$, and then the slides were washed with PBS three times. Images were recorded with a confocal laser scanning microscope (LSM 710 Zeiss, Germany).

\subsection{Fluorescent TUNEL}

Fluorescent TUNEL (terminal deoxynucleotidyl transferase-mediated dUTP nick end labeling assay) was performed with a Fluorescein FragEL DNA Fragmentation Detection Kit (Calbiochem, La Jolla, CA) on frozen retinal sections at $24 \mathrm{~h}$ post-BMSC injection (48 h after ischemia) as we described previously [9], and consistent with the time course of apoptosis after ischemia [19, 20]. Briefly, frozen retinal tissue was fixed and hydrated in $4 \%$ formaldehyde followed by TBS immersion. After permeabilization with proteinase $\mathrm{K}$ (Sigma, St Louis, MO) in $10 \mathrm{mM}$ Tris ( $\mathrm{pH}=8 ; 1: 100)$, tissue was labeled using a TdT enzymatic reaction.

\subsection{Immunohistochemistry}

Enucleated eyes were fixed in $4 \%$ paraformaldehyde at room temperature for $3 \mathrm{~h}$, the anterior segment, and the posterior portion of the eye post-fixed in the same fixative overnight at $4^{\circ} \mathrm{C}$ before being placed in $25 \%$ sucrose for a second overnight period at $4{ }^{\circ} \mathrm{C}$ for cryoprotection. Eyecups were embedded in OCT compound (Sakura Finetec, Torrance, CA) and cut into 10- $\mu \mathrm{m}$ thick sections. Primary antibodies (1:50) were: anti-LC3-II (MBL International, Japan), anti-LAMP (Abcam, Cambridge, MA), and biotin-conjugated mouse monoclonal anti-Thy-1 (BD-Pharmingen, San Jose, CA). Sections were exposed to the appropriate secondary antibodies: goat anti-mouse IgG FITC-conjugate (1:500; Southern Biotechnology, Birmingham, AL), or goat anti-rabbit IgG fluorescein-conjugate (1:500; Invitrogen, Carlsbad, CA). Anti-fade mounting media containing DAPI (EMC Biosciences, La Jolla, CA) was applied and sections cover-slipped. 


\subsection{Western blotting for autophagy and inflammatory markers in retina}

Retinal tissue was homogenized using a tissue homogenizer (Beadbug, Benchmark, Edison, NJ) with RIPA buffer (Cell Signaling Technology, Danvers, MA) containing protease and phosphatase inhibitors. Lysates were centrifuged at $4^{\circ} \mathrm{C}$ and protein concentrations measured using a BCA protein assay kit (Pierce, Rockford, IL) Equal amounts of protein $(40 \mu \mathrm{g})$ were loaded onto $10 \%$ sodium dodecyl sulfate-polyacrylamide gel electrophoresis gels, and Western blotting was performed as we previously reported [16]. The band densities were determined by densitometry with ImageJ (https://imagej.nih.gov/ij/). Antibodies were antiLC3 (to detect LC3-I and LC3-II, MBL International) and p62 (Sigma, St. Louis, MO) for autophagy [21, 22], and anti-TNFa, IL-1 $\beta$, IL-6, and VEGF (Cell Signaling) for inflammatory markers [23]. Band density was normalized to $\beta$-arrestin (Pierce).

\subsection{Blood-retinal barrier permeability}

To evaluate the effect of BMSCs on retinal vascular permeability, we performed an Evans blue dye (EBD) extravasation assay [24]. Seven days post-ischemia and injection of BMSCs, rats were injected with EBD (Sigma-Aldrich, $45 \mathrm{mg} / \mathrm{kg}$ ) via the tail vein, 90 minutes before euthanasia and retina harvesting. The pulmonary circulation was flushed and retinas were harvested. Retinal tissue was dried using a vacuum drier and then weighed. Formamide extracts were prepared by centrifuging homogenates at $12,000 \times g$. Fluorescence in supernatants was measured at 620 and $740 \mathrm{~nm}$. EBD concentration is expressed as $\mu \mathrm{g} / \mathrm{g}$ dry weight of retinal tissue.

\subsection{Imaging and image analysis}

For TUNEL and immunostaining, images were captured with a 40x oil-immersion lens using an Olympus DSU spinning disk confocal microscope (Olympus, Center Valley, PA), and photographed with an Evolve EM-CCD camera (Photometrics, Tucson, AZ) controlled by SlideBook v5.0 (Intelligent Imaging Innovations, Denver, CO). Excitation/dichroic/ emission settings were $405 \mathrm{~nm}$ for DAPI (blue), $488 \mathrm{~nm}$ for FITC (fluorescein/greens), and $640 \mathrm{~nm}$ for rhodamine (red).

\subsection{Data handling}

The ERG a-, b-, OPs and P2-waves from ischemic eyes 7 days after ischemia in the groups for comparison were expressed as normalized intensity-response plots with stimulus intensity $\left(\log \mathrm{cd} \cdot \mathrm{s} \cdot \mathrm{m}^{-2}\right.$ ) on the $\mathrm{x}$-axis, and corresponding percent recovery of the baseline on the $y$-axis, as we previously reported in multiple publications (for example and details, see [25]). Specifically, to account for variation in the electroretinogram amplitudes (that is, including the known day-to-day variation within animals), values obtained for follow-up examinations after ischemia ended were corrected by dividing the normalized ischemic value by the normalized control value (control electroretinogram amplitude at a given time point divided by the baseline control), as we have earlier reported. This analysis corrects for day-to-day expected variation in amplitudes of the ERG waveforms, and for any effects of injection into the control eye. [25] To minimize variability, recordings were made in animals at approximately the same time of day. Recorded amplitude, time course, and intensity were exported and analyzed in Matlab 2011a (The MathWorks, Natick, MA) as previously 
described [16]. As expected, amplitudes of the nSTR and pSTR were low, and were therefore only compared as absolute values.

For stimulus-intensity response ERGs, the a-wave was calculated as the absolute value of the minimum amplitude following the flash stimulus, while the $b$-wave was calculated as the difference between the negative a-wave value and the maximum amplitude recorded thereafter [16]. The OP- and P2-waves were derived as we previously reported [16]. OPvalues were calculated as sum of the root mean squares (Sum RMS) of the amplitudes [16]. For STR [26], there is no conventional stimulus intensity procedure as for the ERG, thus, responses to six flash intensities were evaluated. The lower intensity waveforms were baseline corrected to remove positive or negative drift artifact. The process was to subtract the linear approximation of the original waveform from itself, thereby removing the upward or downward inflection and returning the waveform to the correct baseline level. The comparatively small amplitudes allowed for the linear approximation to be used as a baseline transform. The linear baseline correction generated a more absolute measurement of the positive and negative STR, since the maximum measurements are made from a zero 'baseline' rather than from a non-zero 'tilted baseline.' Positive STR (pSTR) was calculated as maximum positive amplitude, while negative STR (nSTR) was calculated as maximum negative amplitude

\subsection{Statistics}

Data were compared by t-testing (Stata, College Station, TX). Between groups, we used an unpaired t-test and within groups (e.g., normal vs ischemic retina), a paired t-test was used.

\section{Results}

\subsection{Functional neuroprotection by BMSCs}

BMSCs injected $24 \mathrm{~h}$ after ischemia significantly improved the a-wave, b-wave, OP, and P2wave recovery $(n=8-9, p<0.05)$ at 7 days after the injection, when compared to the PBS control group at flash intensities from -3.39 to $1.4 \log \mathrm{cd} \cdot \mathrm{s} \cdot \mathrm{m}^{-2}$ (Fig 1A-D); e.g., at $0.87 \mathrm{log}$ $\mathrm{cd} \cdot \mathrm{s} \cdot \mathrm{m}^{-2}$ the a-wave BMSC recovery was $100.2 \pm 0.2 \%$ versus vehicle $42.7 \pm 0.1 \%$, b-wave BMSC recovery was $59.9 \pm 0.1 \%$ versus $26.3 \pm 0.1 \%$ for the vehicle control, P2 BMSC recovery was $63.1 \pm 0.1 \%$ versus $27.1 \pm 0.1 \%$ for the vehicle control, and OP BMSC recovery was $47.3 \pm 0.1 \%$ versus $19.1 \pm 0.1 \%$ for vehicle control.

For amplitudes of the nSTR and pSTR, there were no significant differences in the control non ischemic eyes (Fig 2A and 2C). The nSTR was better preserved after ischemia with BMSC injection vs vehicle, but pSTR was significantly decreased after ischemia and no different for BMSC vs vehicle (Fig 2B and 2D).

Fig 3 shows effects of injections upon the non-ischemic eye, as well as the amplitudes of the ERG waveforms at each intensity for the non-ischemic and the ischemic eyes. There were no differences between non-ischemic values for the OPRMS (Fig 3E), and few for the a-wave (Fig 3A). For the b-wave (Fig 3C) and P2 (Fig 3D), both injection of BMSCs and PBS vehicle decreased amplitudes of the waveforms, and the vehicle-injected eyes had generally higher baseline amplitudes compared to the BMSC-injected. There was a greater decrease in 
amplitudes of the waveforms from baseline to day 7 in ischemic eyes for all waveforms for the vehicle compared to the BMSC injected eyes (Fig 3E-H), which can be seen more clearly in Fig 1, where effects of injections and measurement time are accounted for simultaneously.

\subsection{BMSCs incorporate into ischemic retina}

To track BMSCs in the retina, we used in vivo imaging of rat eyes at 1,7 and 21 days' post injection, and examined flat mounts of retina injected with GFP-labeled BMSCs at 7 days after injection. Fig 4A displays the fate of GFP-labelled BMSCs injected into the vitreous and their survival in the eye. To examine incorporation of cells into the retinal tissue, flat mounts were prepared and imaged using confocal microscopy. Representative retinal flat mounts from PBS and BMSC injected eyes (Fig 4B) demonstrate qualitatively large numbers of labeled stem cells retained in the retina at 7 days post injection of BMSCs.

\subsection{BMSCs attenuate apoptosis in retinal ischemia}

To test the pro-survival effect of BMSCs in ischemia, we performed fluorescent TUNEL on retinal cryosections (Fig. 5A and B) 24h post-ischemia, a time point consistent with peak apoptosis in our and others' published studies [19, 20]. BMSCs injection $24 \mathrm{~h}$ after ischemia significantly reduced \% TUNEL in the RGC layer (PBS-injected, $30.3 \pm 6.7 \%$ vs BMSCstreated ischemic retinae, $12.0 \pm 3.5 \%, \mathrm{n}=7 ; \mathrm{p}<0.05$ ). Double labeling with anti-thy 1 for RGCs [27], confirmed TUNEL was in the RGCs of the ischemic retina (Fig 5C).

Additionally, a significant reduction in cleaved caspase-3 level in BMSC injected ischemic retinal lysates vs PBS ischemic-injected ( $\mathrm{n}=5$, Fig 5D) further supports the presence of apoptotic cell death and its attenuation by BMSCs in ischemic retinae.

\subsection{BMSCs transplantation reduced neuro-inflammation and vascular permeability}

Western blot analysis exhibited the anti-inflammatory effect of BMSCs as demonstrated by attenuated levels of inflammatory markers in the ischemic retina treated with BMSCs. Consistent with previous studies, levels of pro-inflammatory markers TNF-a, IL-1 $a$ and Il-6 were elevated in retina subjected to ischemia $(n=3-4)$ [23]. BMSCs attenuated these increases in inflammatory mediators (Fig 6A-C). However, there was an increase in VEGF in the BMSC group vs PBS control (Fig 6D).

Quantitation of extravasated Evans Blue (EB) in the formamide extracts from PBS and BMSC injected ischemic retina illustrated remarkable vascular barrier protection in BMSCtreated ischemic retina as evidenced by significantly decreased EB in BMSC injected eyes compared to PBS control group $(n=7$, Fig $6 E)$.

\subsection{Autophagy is involved in BMSC induced protection from retinal ischemic injury}

Levels of autophagy- related proteins LC3 and p62, were investigated. Relative to the normal non-ischemic eye, LC3-I decreased, and LC3-II was significantly increased, in BMSC injected ischemic retina vs PBS (Fig 7A), while p62 decreased $(\mathrm{n}=5)$. Increased LC3-II with decreased P62 suggests enhanced autophagic flux [28]. To confirm LC3-II labeling of autophagosomes, a hallmark of autophagy [29], we immunostained for LC3-II and LAMP; the latter is found in autophagosomes [30]. LC3-II and LAMP co-localized in 
the inner nuclear and retinal ganglion cell layer; double labeled LC3-II and LAMP was present in the cytoplasm of cells in the retina, in particular in the RGC layer, confirming the presence of autophagosomes (Fig 7B).

\section{Discussion}

We examined the effect of delayed administration of autologous bone marrow-derived mesenchymal stem cell transplantation in our rat model of retinal ischemia. Our results demonstrate that injection of BMSCs $24 \mathrm{~h}$ post-ischemia significantly protected the retina from damage as illustrated by improved function, decreased retinal inflammation and vascular permeability, and enhanced autophagy.

Stem cell therapy faces challenges of cell survival and differentiation, but conversely, excessive proliferation and aberrant differentiation. Although some studies reported incorporation of BMSCs and differentiation into neuronal cells [10], the majority of the cells remained in the vitreous after intravitreal injection, posing a limiting factor in retinal stem cell therapy. In a retinal explant model, Johnson et al demonstrated that glial reactivity constituted the major barrier to migration of BMSCs into the retina [31]. Similarly, in a mouse model of diabetes, intra-vitreally injected MSCs remained in the vitreous, but they increased the levels of neurotrophic factors and reduced oxidative damage.[12] It is therefore interesting that in our study, GFP-tagged BMSCs entered ischemic retina. The migration of BMSCs into the post-ischemic retina may be possible due to the delayed time course of gliosis following retinal ischemia, because in our study, BMSCs were injected prior to the influence of enhanced glial reactivity. Muller cells in retinal slices taken 3 days after ischemia from porcine eyes had significant swelling of the somata when subjected to osmotic stress from hypotonic extracellular solution.[32] In ischemic rabbit eyes, ischemia caused exudative detachment of the central retina with disruption of the pigment epithelial monolayer, scattered pigment epithelial and immune cells in the sub-retinal space, and retinal folds. Muller cell gliosis was not apparent until 3-8 days later. [33]

Our previous studies on ischemic preconditioning [13, 15, 34, 35], post-ischemic conditioning [36], injection of BMSC conditioned media [16], or hypoxic preconditioned media [9], reported attenuation of apoptosis as a mechanism of protection from retinal ischemia. Here we demonstrate that BMSCs induced protective effects are also in part, due to reduced inner retinal cell apoptotic death. The most profound functional effects in this study were found in the b wave, P2, and the STR. These parameters reflect activity of cells in the inner retina, the rod bipolar cells, and the retinal ganglion and amacrine cells, and are consistent with the TUNEL findings in this study as in others, that the inner retina is relatively preserved after ischemia by BMSC injection.

In our previous studies, normoxic, and hypoxic-preconditioned BMSC media produced 4fold improvement in recovery of the $\mathrm{b}$ wave and $\mathrm{P} 2$ after ischemia vs approximately 2 -fold in the present study; the recovery after hypoxic-preconditioned BMSC media was about 80$90 \%$ of baseline, thus, nearly complete. Using both modalities, BMSCs or media, post ischemic apoptotic cell death was attenuated. It is possible that the more effective recovery with media was due to more effective delivery (less dilution) of the BMSC secretome 
compared to injecting BMSCs. Another possible explanation is death of the BMSCs over time after injection, and thus less delivery of secretome. Comparing BMSC media to BMSCs themselves, there are pros and cons of each approach. Cells, provided they survive, could potentially release neuroprotective factors for more extended periods of time vs media. However, media likely results in higher levels of secretome and better penetration into retina, but, without encapsulation, release of neuroprotective factors is time-limited. There are also potential complications of BMSC injection (see end of Discussion for details).

We showed in this study other ameliorative mechanisms of BMSCs. Inflammation is a consistent feature of ischemia, with augmented cytokines and chemokines in the retina [37], significantly attenuated in BMSC-injected ischemic retinae. Anti-inflammatory benefits of BMSCs were reported via direct interaction with endogenous cells or release of paracrine factors [38]. Retina responds to ischemia-induced oxidative stress with increased inflammation and vascular permeability $[4,39]$. Studies on rats subjected to ischemiareperfusion, reported vascular leakage with EBD dye extravasation from the retinal vasculature [4]. The mechanisms of attenuation of the blood-retinal barrier by BMSCs were not determined in the present study. However, some stem cells have been found to produce genes mediating barrier properties [40], and adipose-derived stem cells prevented bloodretinal barrier leakage in diabetic rats possibly via differentiation into glial cells [41].

We found that VEGF levels were increased about 3-fold in BMSC transplanted retina. Because the levels were measured as relative changes by Western blotting, and not absolutely, we cannot state what the concentration of VEGF was in the retina, and how it compares to physiological levels. In retinal ischemia, VEGF decreases in a gradient from the RGC to the outer plexiform layer. With rapid damage to the RGCs, neuronal cell damage precedes capillary degeneration, Endothelial cell survival factors, including VEGF, are released by retinal neuronal cells, including RGCs [42, 43]. Thus another potential mechanism for enhanced survival of the retina in our study is the stimulation of VEGF production by retinal neurons.

Increased LC-3II, decreased P62, and co-labeling of LAMP and LC3-II in retinal cells, the latter proving the presence of autophagosomes, suggest that BMSCs increased autophagic flux in ischemic retinae [28]. The relationship of autophagy to retinal survival after ischemia remains controversial, with some groups demonstrating that increased autophagy enhances, and others showing it decreases survival $[44,45]$. Complicating the effects, autophagy can be induced in BMSCs to either improve or decrease resistance to hypoxia [46]. Thus, it remains suggestive but not proven, that autophagy was protective against ischemic damage in the present study.

Retinal cross-talk has been recently reported. In Brown Norway rats, monocular light flashes produced a signal in the non-light flashed eye, the "crossed ERG" (xERG). Intraocular pressure elevation of the flashed eye eliminated the xERG. Due to its attenuation by elevated IOP and very small magnitude (10-15 $\mu \mathrm{v})$, it is improbable that the xERG, or by inference, an effect of injecting the non-ischemic eye, played any role our results with respect to the a, b, OP waves or the P2, or the STR, all of which were recorded under entirely different conditions in our study [47] 
Our current results highlight a potent and clinically important procedure by which to prevent ischemic retinal damage. Transplantation of the BMSCs may be a longer-lasting solution vs administration of media, because of persistence of the cells in the vitreous. However, there are limitations of this approach. The persistence of cells in the vitreous may diminish visual acuity. Immune reactions, retinal detachment, and tumor transformation are also potential complications [7]. Limitations of the study, overall, are that the rodent retina lacks a macula, IOP elevation may not resemble central retinal artery occlusion, and the outcomes were studied only out to 7 days after ischemia. BMSCs are readily obtained and we demonstrated several mechanisms whereby the stem cells decrease ischemic injury, providing support for human trials of BMSC treatment in ischemic retina.

\section{Acknowledgments}

Funding:

This study was supported by National Institutes of Health (Rockville, MD) grants EY10343 and EY10343-16S1 (American Recovery and Reinvestment Act) to Dr. Roth, NS087990 to Dr. Lesniak and to Dr. Balyasnikova, AG029795 for the Medical Student Summer Research Program at the University of Chicago Pritzker School of Medicine, UL1RR024999 to the University of Chicago Institute for Translational Medicine; the Illinois Society for the Prevention of Blindness, Chicago, IL (Dr. Poston); Core Grant P30 EY001792 (to the Department of Ophthalmology, University of Illinois at Chicago, Chicago, Illinois); and a Center-Style Grant from the Dean's Research Advisory Committee of the Division of Biological Sciences of the University of Chicago (Drs. Lesniak and Roth). Jacqueline N. Poston was the recipient of a Medical Student Research Fellowship Award from the American Academy of Neurology (St. Paul, Minnesota, USA) and a student scholarship from the Achievement Rewards for College Scientists Foundation (Washington, DC, USA). There was no involvement of the funding bodies in the design of the study or in collection, analysis and interpretation of the data or the writing of the manuscript.

\section{Abbreviations}

BMSC Bone marrow mesenchymal stem cells

ERG electroretinogram

EBD Evans blue dye extravasation

GFP-BMSCgreen fluorescent-labeled BMSCs

IR ischemia-reperfusion

RGCs retinal ganglion cells

STR scotopic threshold response

\section{References}

1. Al-Shabrawey M, Rojas M, Sanders T, Behzadian A, El-Remessy A, Bartoli M, Parpia AK, Liou G, Caldwell RB. Role of NADPH oxidase in retinal vascular inflammation. Investigative Ophthalmology \& Visual Science. 2008; 49:3239-3244. [PubMed: 18378574]

2. Hangai M, Yoshimura N, Honda Y. Increased cytokine gene expression in rat retina following transient ischemia. Ophthalmic Research. 1996; 28:248-254. [PubMed: 8878188]

3. Gustavsson C, Agardh C-D, Hagert P, Agardh E. Inflammatory markers in nondiabetic and diabetic rat retinas exposed to ischemia followed by reperfusion. Retina. 2008; 28:645-652. [PubMed: 18398369] 
4. Abcouwer SF, Lin CM, Wolpert EB, Shanmugam S, Schaefer EW, Freeman WM, Barber AJ, Antonetti DA. Effects of ischemic preconditioning and bevacizumab on apoptosis and vascular permeability following retinal ischemia-reperfusion injury. Investigative Ophthalmology \& Visual Science. 2010; 51:5920-5933. DOI: 10.1167/iovs.10-5264 [PubMed: 20554620]

5. Osborne NN, Casson RJ, Wood JPM, Chidlow G, Graham M, Melena J. Retinal ischemia: mechanisms of damage and potential therapeutic strategies. Progress in Retinal \& Eye Research. 2004; 23:91-147. [PubMed: 14766318]

6. Dattilo M, Biousse V, Newman NJ. Update on the Management of Central Retinal Artery Occlusion. Neurologic Clinics. 2017; 35:83-100. DOI: 10.1016/j.ncl.2016.08.013 [PubMed: 27886897]

7. Park SS, Moisseiev E, Bauer G, Anderson JD, Grant MB, Zam A, Zawadzki RJ, Werner JS, Nolta JA. Advances in bone marrow stem cell therapy for retinal dysfunction. Progress in Retinal and Eye Research. 2017; 56:148-165. DOI: 10.1016/j.preteyeres.2016.10.002 [PubMed: 27784628]

8. Sun J, Wei ZZ, Gu X, Zhang JY, Zhang Y, Li J, Wei L. Intranasal delivery of hypoxiapreconditioned bone marrow-derived mesenchymal stem cells enhanced regenerative effects after intracerebral hemorrhagic stroke in mice. Experimental Neurology. 2015; 272:78-87. DOI http:// dx.doi.org/10.1016/j.expneurol.2015.03.011. [PubMed: 25797577]

9. Roth S, Dreixler JC, Mathew B, Balyasnikova I, Mann JR, Boddapati V, Xue L, Lesniak MS. Hypoxic-Preconditioned Bone Marrow Stem Cell Medium Significantly Improves Outcome After Retinal Ischemia in RatsPreconditioned Stem Cells and Retinal Ischemia. Investigative Ophthalmology \& Visual Science. 2016; 57:3522-3532. DOI: 10.1167/iovs.15-17381 [PubMed: 27367588]

10. Li N, Li X-r, Yuan J-q. Effects of bone-marrow mesenchymal stem cells transplanted into vitreous cavity of rat injured by ischemia/reperfusion. Graefes Archiv of Clinical and Experimental Ophthalmology. 2009; 247:503-514.

11. Zheng L, Gong B, Hatala DA, Kern TS. Retinal ischemia and reperfusion causes capillary degeneration: similarities to diabetes. Investigative Ophthalmology \& Visual Science. 2007; 48:361-367. [PubMed: 17197555]

12. Dowling JE. NEURAL AND PHOTOCHEMICAL MECHANISMS OF VISUAL ADAPTATION IN THE RAT. The Journal of General Physiology. 1963; 46:1287-1301. [PubMed: 14043003]

13. Roth S, Shaikh AR, Hennelly MM, Li Q, Bindokas V, Graham CE. Mitogen-activated protein kinases and retinal ischemia. Investigative Ophthalmology \& Visual Science. 2003; 44:5383-5395. [PubMed: 14638742]

14. Roth S, Dreixler JC, Shaikh AR, Lee KH, Bindokas V. Mitochondrial potassium ATP channels and retinal ischemic preconditioning. Investigative Ophthalmology \& Visual Science. 2006; 47:21142124. [PubMed: 16639023]

15. Dreixler JC, Shaikh AR, Shenoy SK, Shen Y, Roth S. Protein kinase C subtypes and retinal ischemic preconditioning. Experimental Eye Research. 2008; 87:300-311. [PubMed: 18722601]

16. Dreixler JC, Poston JN, Balyasnikova I, Shaikh AR, Lesniak MS, Roth S. Delayed administration of bone marrow mesenchymal stem cell conditioned medium significantly improves outcome after retinal ischemia in Rats. Investigative Ophthalmology \& Visual Science. 2014; 55:3785-3796. DOI: 10.1167/iovs.13-11683 [PubMed: 24699381]

17. Weymouth AE, Vingrys AJ. Rodent electroretinography: methods for extraction and interpretation of rod and cone responses. Progress in Retinal and Eye Research. 2008; 27:1-44. [PubMed: 18042420]

18. Bui BV, Edmunds B, Cioffi GA, Fortune B. The gradient of retinal functional changes during acute intraocular pressure elevation. Investigative Ophthalmology \& Visual Science. 2005; 46:202-213. [PubMed: 15623775]

19. Singh M, Savitz SI, Hoque R, Rosenbaum PS, Roth S, Rosenbaum DM. Cell-specific caspase expression by different neuronal phenotypes in transient retinal ischemia. Journal of Neurochemistry. 2001; 77:466-475. [PubMed: 11299309]

20. Zhang C, Rosenbaum DM, Shaikh AR, QL, Rosenbaum PS, Pelham DJ, Roth S. Ischemic preconditioning attenuates apoptosis following retinal ischemia in rats. Investigative Ophthalmology \& Visual Science. 2002; 43:3059-3066. [PubMed: 12202530] 
21. Huang C, Andres AM, Ratliff EP, Hernandez G, Lee P, Gottlieb RA. Preconditioning involves selective mitophagy mediated by Parkin and p62/SQSTM1. PloS One. 2011; 6:e20975.doi: 10.1371/journal.pone.0020975 [PubMed: 21687634]

22. Carloni S, Girelli S, Scopa C, Buonocore G, Longini M, Balduini W. Activation of autophagy and Akt/CREB signaling play an equivalent role in the neuroprotective effect of rapamycin in neonatal hypoxia-ischemia. Autophagy. 2015; 6:366-377.

23. Fang IM, Yang C-M, Yang C-H, Chiou S-H, Chen M-S. Transplantation of induced pluripotent stem cells without C-Myc attenuates retinal ischemia and reperfusion injury in rats. Experimental Eye Research. 2013; 113:49-59. DOI http://dx.doi.org/10.1016/j.exer.2013.05.007. [PubMed: 23726881]

24. Goncalves A, Lin CM, Muthusamy A, Fontes-Ribeiro C, Ambrosio AF, Abcouwer SF, Fernandes R, Antonetti DA. Protective Effect of a GLP-1 Analog on Ischemia-Reperfusion Induced BloodRetinal Barrier Breakdown and Inflammation. Investigative Ophthalmology \& Visual Science. 2016; 57:2584-2592. DOI: 10.1167/iovs.15-19006 [PubMed: 27163772]

25. Dreixler JC, Poston JN, Shaikh AR, Alexander M, Tupper KY, Marcet MM, Bernaudin M, Roth S. Delayed post-ischemic conditioning significantly improves the outcome after retinal ischemia. Experimental Eye Research. 2011; 92:521-527. [PubMed: 21501608]

26. Fortune B, Bui BV, Morrison JC, Johnson EC, Dong J, Cepurna WO, Jia L, Barber S, Cioffi GA. Selective ganglion cell functional loss in rats with experimental glaucoma. Investigative Ophthalmology \& Visual Science. 2004; 45:1854-1862. [PubMed: 15161850]

27. Schlamp CL, Johnson EC, Li Y, Morrison JC, Nickells RW. Changes in Thy 1 gene expression associated with damaged retinal ganglion cells. Molecular Vision. 2001; 7:192-201. [PubMed: 11509915]

28. Tanida I, Minematsu-Ikeguchi N, Ueno T, Kominami E. Lysosomal turnover, but not a cellular level, of endogenous LC3 is a marker for autophagy. Autophagy. 2005; 1:84-91. [PubMed: 16874052]

29. Feng Y, Yao Z, Klionsky DJ. How to control self-digestion: transcriptional, post-transcriptional, and post-translational regulation of autophagy. Trends in Cell Biology. 2015; doi: 10.1016/j.tcb. 2015.02.002

30. Saftig P, Beertsen W, Eskelinen EL. LAMP-2: a control step for phagosome and autophagosome maturation. Autophagy. 2008; 4:510-512. [PubMed: 18376150]

31. Johnson TV, Bull ND, Martin KR. Identification of barriers to retinal engraftment of transplanted stem cells. Investigative Ophthalmology \& Visual Science. 2010; 51:960-970. [PubMed: 19850833]

32. Reichenbach A, Wurm A, Pannicke T, Iandiev I, Wiedemann P, Bringmann A. Muller cells as players in retinal degeneration and edema. Graefes Archive for Clinical \& Experimental Ophthalmology. 2007; 245:627-636.

33. Uckermann O, Kutzera F, Wolf A, Pannicke T, Reichenbach A, Wiedemann P, Wolf S, Bringmann A. The glucocorticoid triamcinolone acetonide inhibits osmotic swelling of retinal glial cells via stimulation of endogenous adenosine signaling. Journal of Pharmacology \& Experimental Therapeutics. 2005; 315:1036-1045. [PubMed: 16144977]

34. Dreixler JC, Barone FC, Shaikh AR, Du E, Roth S. Mitogen-activated protein kinase p38alpha and retinal ischemic preconditioning. Experimental Eye Research. 2009; 89:782-790. [PubMed: 19631642]

35. Dreixler JC, Hemmert JW, Shenoy SK, Shen Y, Lee HT, Shaikh AR, Rosenbaum DM, Roth S. The role of Akt/protein kinase B subtypes in retinal ischemic preconditioning. Experimental Eye Research. 2009; 88:512-521. [PubMed: 19084003]

36. Dreixler J, Shaikh A, Alexander M, Savoie B, Roth S. Post-ischemic conditioning in the rat retina is dependent upon ischemia duration and is not additive with ischemic preconditioning.

Experimental Eye Research. 2010; 91:844-852. [PubMed: 20599964]

37. Jo N, Wu G-S, Rao NA. Upregulation of chemokine expression in the retinal vasculature in ischemia-reperfusion injury. Investigative Ophthalmology \& Visual Science. 2003; 44:4054-4060. [PubMed: 12939328] 
38. Hattori T, Hayashi H, Chiba T, Onozaki K. Activation of two distinct anti-proliferative pathways, apoptosis and p38 MAP kinase-dependent cell cycle arrest, by tumor necrosis factor in human melanoma cell line A375. European Cytokine Network. 2001; 12(2):244-52. [PubMed: 11399512]

39. Danesh-Meyer HV, Kerr NM, Zhang J, Eady EK, O'Carroll SJ, Nicholson LFB, Johnson CS, Green CR. Connexin43 mimetic peptide reduces vascular leak and retinal ganglion cell death following retinal ischaemia. Brain. 2012; 135:506-520. DOI: 10.1093/brain/awr338 [PubMed: 22345088]

40. Peng J, Drobish JK, Liang G, Wu Z, Liu C, Joseph DJ, Abdou H, Eckenhoff MF, Wei H. Anesthetic preconditioning inhibits isoflurane-mediated apoptosis in the developing rat brain. Anesthesia and Analgesia. 2014; 119:939-946. DOI: 10.1213/ane.0000000000000380 [PubMed: 25099925]

41. Feng Y, Rhodes PG, Bhatt AJ. Hypoxic preconditioning provides neuroprotection and increases vascular endothelial growth factor A, preserves the phosphorylation of Akt-Ser-473 and diminishes the increase in caspase- 3 activity in neonatal rat hypoxic-ischemic model. Brain Research. 2015; 1325:1-9.

42. Amato R, Biagioni M, Cammalleri M, Dal Monte M, Casini G. VEGF as a Survival Factor in Ex Vivo Models of Early Diabetic Retinopathy. Investigative Ophthalmology \& Visual Science. 2016; 57:3066-3076. DOI: 10.1167/iovs.16-19285 [PubMed: 27286364]

43. Nakahara T, Hoshino M, Hoshino S, Mori A, Sakamoto K, Ishii K. Structural and functional changes in retinal vasculature induced by retinal ischemia-reperfusion in rats. Experimental Eye Research. 2015; 135:134-145. DOI: 10.1016/j.exer.2015.02.020 [PubMed: 25728136]

44. Piras A, Gianetto D, Conte D, Bosone A, Vercelli A. Activation of autophagy in a rat model of retinal ischemia following high intraocular pressure. PloS One. 2011; 6:e22514.doi: 10.1371/ journal.pone.0022514 [PubMed: 21799881]

45. Russo R, Berliocchi L, Adornetto A, Varano GP, Cavaliere F, Nucci C, Rotiroti D, Morrone LA, Bagetta G, Corasaniti MT. Calpain-mediated cleavage of Beclin-1 and autophagy deregulation following retinal ischemic injury in vivo. Cell Death \& Disease. 2011; 2:e144.doi: 10.1038/cddis. 2011.29 [PubMed: 21490676]

46. Wang G, Zhou D, Wang C, Gao Y, Zhou Q, Qian G, DeCoster MA. Hypoxic preconditioning suppresses group III secreted phospholipase A2-induced apoptosis via JAK2-STAT3 activation in cortical neurons. Journal of Neurochemistry. 2010; 114:1039-1048. DOI: 10.1111/j. 1471-4159.2010.06817.x [PubMed: 20492356]

47. Tang X, Tzekov R, Passaglia CL. Retinal cross talk in the mammalian visual system. Journal of Neurophysiology. 2016; 115:3018-3029. DOI: 10.1152/jn.01137.2015 [PubMed: 26984426] 
A.

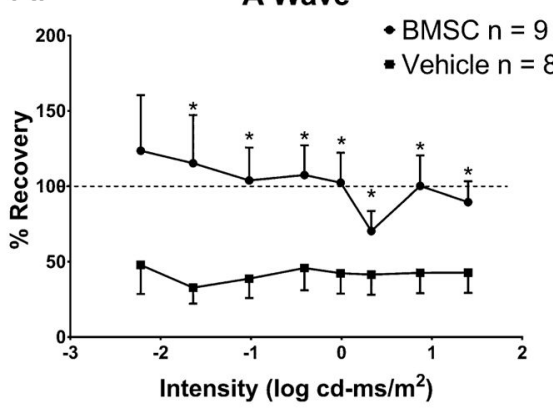

C.

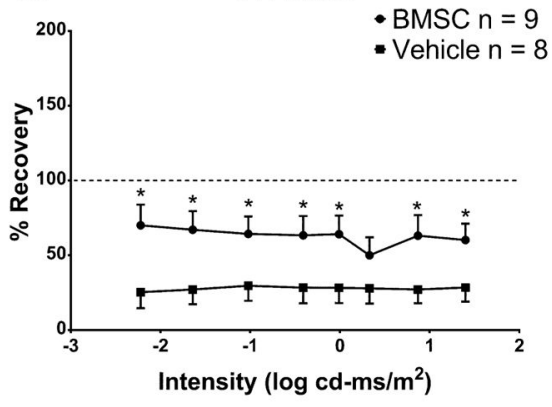

B.

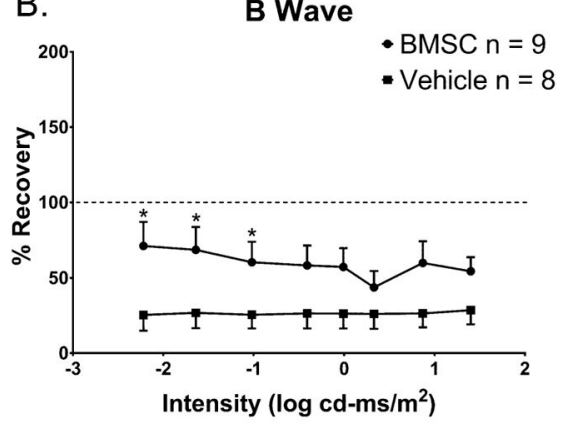

D.

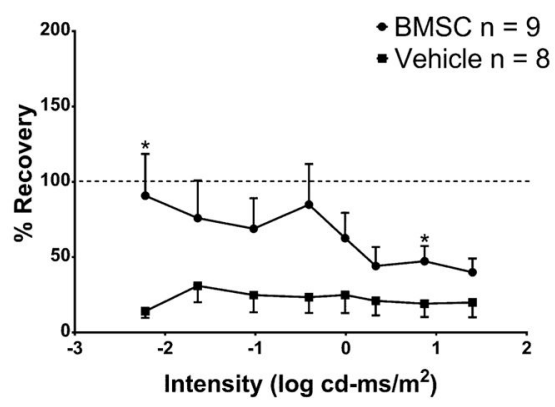

Figure 1.

Stimulus-Intensity responses for the a-wave (A), b-wave (B), P2 (C), sum of the root mean squared OP waves (OPRMS) (D) from electroretinograms in rats subjected to retinal ischemia for $55 \mathrm{~min}$ and the eyes injected $24 \mathrm{~h}$ later with BMSCs or control (PBS). The recovery was measured 7 days later; $\mathrm{N}=8-9$. Normalized ERG recovery data (Y-axis, see Methods) for the waves over a range of up to 10 flash intensities (x-axis) are shown. For the a-wave, data collection began at a higher initial flash intensity due to artifact and low amplitude at the two lower intensities. There was significant improvement with injection of BMSCs $24 \mathrm{~h}$ after ischemia on all of the ERG waves, although results were not statistically significant at all intensity levels. Data are shown as mean \pm SEM. $* \mathrm{P}<0.05$ vs PBS vehicle control. Representative ERG traces for a- and b-waves, OP, and P2 from our lab are available in previous publications [16]. 

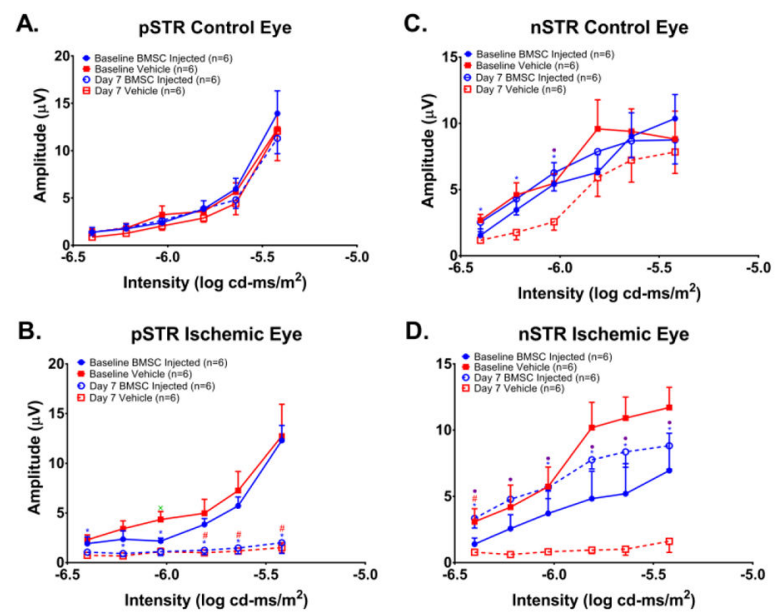

D.
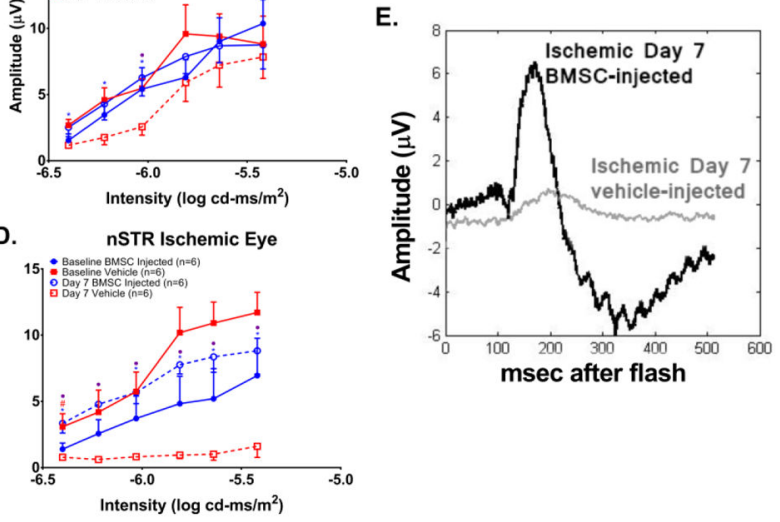

Figure 2.

(A\&C) Stimulus-Intensity responses for the STR (scotopic threshold response), for the positive STR (pSTR) and negative STR (nSTR) in non-ischemic eyes of rats subjected to retinal ischemia for $55 \mathrm{~min}$ and the eyes injected $24 \mathrm{~h}$ later with BMSCs or control (PBS). (B\&D) STRs recorded from the ischemic eyes at baseline and after ischemia. The recovery was measured 7 days later. Absolute amplitude appears on the Y-axis (see Methods) and the 6 flash intensities are on the X-axis. There was significant improvement in the nSTR, but not the pSTR with injection of BMSCs $24 \mathrm{~h}$ after ischemia. Data are shown as mean $+\mathrm{SEM} . * \mathrm{P}$ $<0.05$ vehicle Day 7 vs baseline; \# $=\mathrm{P}<0.05$ BMSC Day 7 vs baseline; $\mathrm{x}=\mathrm{P}<0.05$ vehicle baseline vs BMSC baseline;. $=\mathrm{P}<0.05$ vehicle Day 7 vs BMSC day 7. $\mathrm{N}=6$. (E) Representative STR traces at the highest flash intensity, with BMSC-ischemic eye on top, and PBS-ischemic eye below 

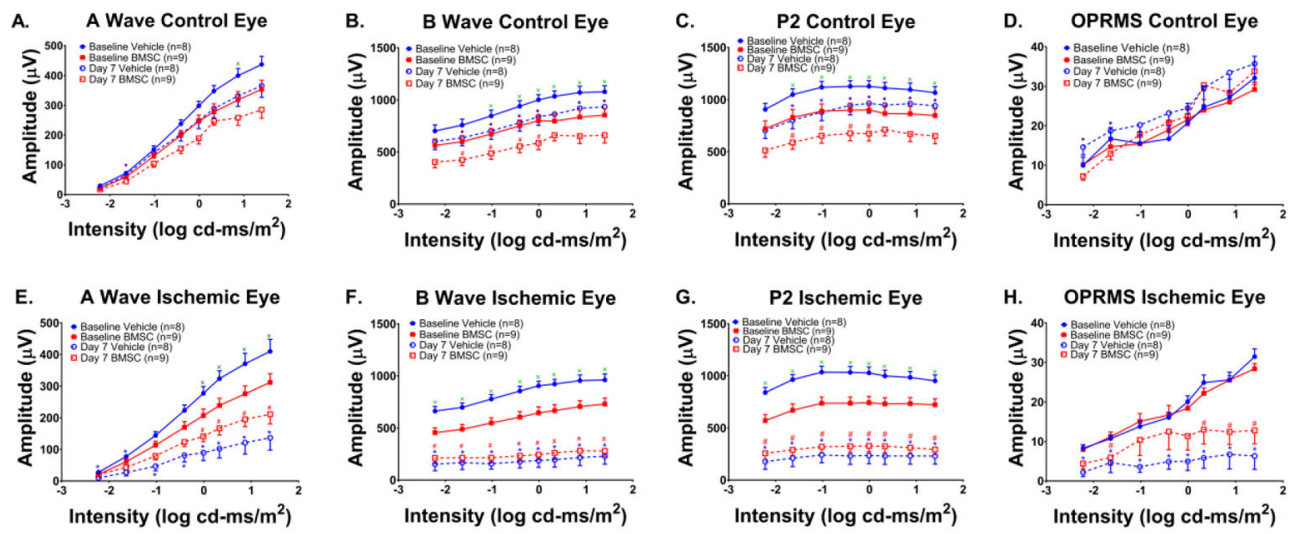

Figure 3.

(A, C, G, E) Stimulus-Intensity responses for the a wave, b-wave, P2, and OPRMS in nonischemic eyes of rats subjected to retinal ischemia for $55 \mathrm{~min}$ and the eyes injected $24 \mathrm{~h}$ later with BMSCs or control (PBS). (B, D, F, H) Stimulus intensity responses for a, b, P2, and OPRMS waves recorded from the ischemic eyes at baseline and after ischemia. The recovery was measured 7 days later. Absolute amplitude appears on the Y-axis (see Methods) and the flash intensities are on the X-axis. Data are shown as mean + SEM. There were no differences between non-ischemic values for the OPRMS (Fig 3E), and few for the a-wave. For the b-wave and P2, both injection of BMSCs and PBS vehicle decreased amplitudes of the waveforms, and the vehicle-injected eyes had generally higher baseline amplitudes compared to the BMSC-injected. There was a greater decrease in amplitudes of the waveforms from baseline to day 7 in ischemic eyes for all waveforms for the vehicle compared to the BMSC injected eyes. * $\mathrm{P}<0.05$ vehicle Day 7 vs baseline; $\#=\mathrm{P}<0.05$ BMSC Day 7 vs baseline; $\mathrm{x}=\mathrm{P}<0.05$ vehicle baseline vs BMSC baseline; $=\mathrm{P}<0.05$ vehicle Day 7 vs BMSC day $7 . \mathrm{N}=8-9$ 
A. Time post BMSC injection

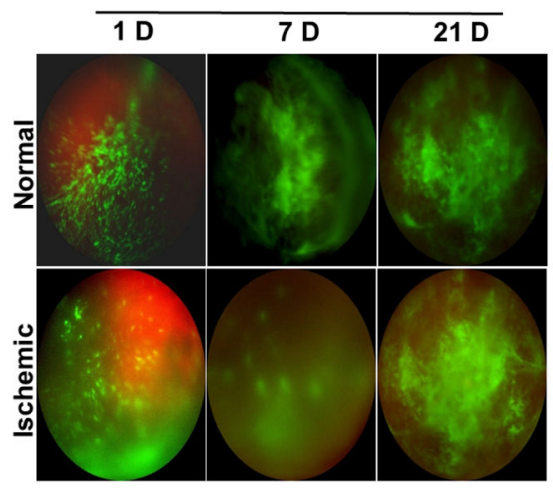

B. Day 7 post BMSC injection-ischemic eye

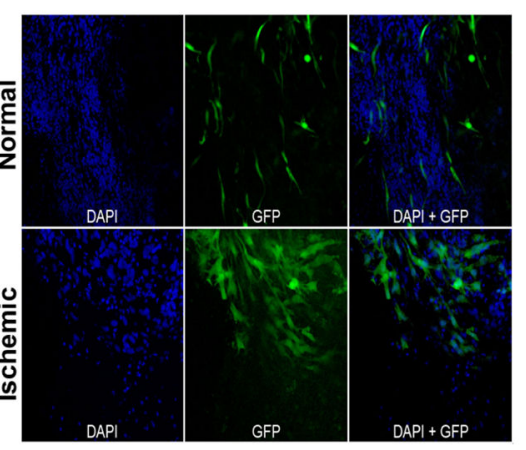

Figure 4.

Ischemic and normal eyes were injected with GFP-BMSC $24 \mathrm{~h}$ post ischemia. In vivo imaging of the eyes was performed at 1, 7 and 21 days' post injection (A). The upper and lower panels exhibit representative images of normal and ischemic eyes injected with BMSCs. (B) Representative flat mount images of BMSCs injected retina illustrate the penetration and incorporation of significantly increased number of GFP-BMSCs into the ischemic retinal tissue 7 days' post injection compared to normal retina. DAPI was used to stain nuclei. 


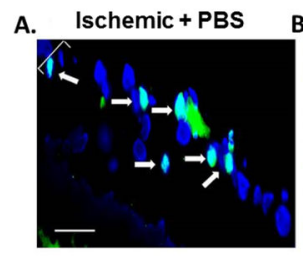

C.

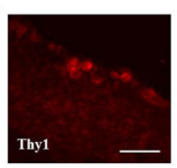

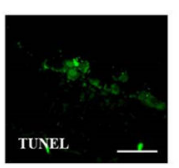
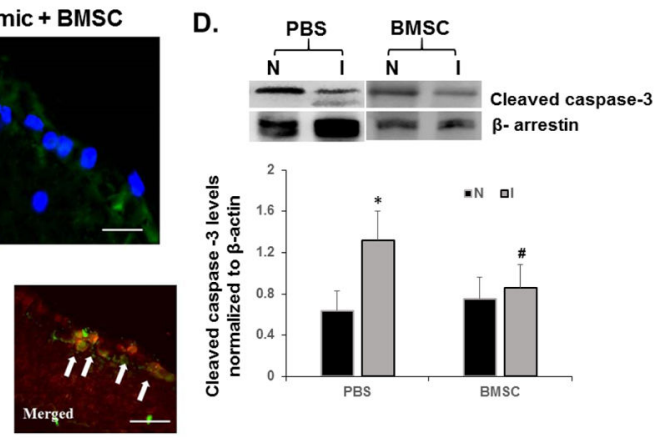

Figure 5.

Fluorescent TUNEL staining for evaluation of apoptosis in $10-\mu \mathrm{m}$ thick retinal cryosections: BMSC injection into the retina significantly reduced apoptotic cell death in the ischemic retinae $24 \mathrm{~h}$ post injection (B) as compared to PBS injected ischemic control (A). White arrows denote TUNEL positive cells (green) co-localized with DAPI (blue). Brackets denote the retinal ganglion cell layer. Magnification $=40 \mathrm{X}$. Scale bar $=15 \mu \mathrm{m}$. $(\mathbf{C})$ Retinal ganglion cells undergoing apoptosis in PBS injected control ischemic retina identified by colocalization of TUNEL and Thy1 (retinal ganglion cell marker, red). Magnification $=40 \mathrm{X}$. Scale bars $=20 \mu \mathrm{m}$. (D) Representative Western blot images for cleaved caspase-3 in retinal lysates obtained from PBS or BMSC ischemic injected eyes and paired normal eyes, at $24 \mathrm{~h}$ after injection of PBS or BMSCs. Levels of cleaved caspase- 3 were normalized to $\beta$-arrestin. Data are shown as mean \pm SEM. * indicates $\mathrm{P}<0.05$ for ischemic compared to the paired normal retina within a group (PBS or BMSC); \# indicates $\mathrm{P}<0.05$ between the ischemic retinae of PBS and BMSC. $\mathrm{N}=$ normal, non-ischemic retinae, and $\mathrm{I}=$ ischemic retinae. $\mathrm{N}=$ 5 per group. 


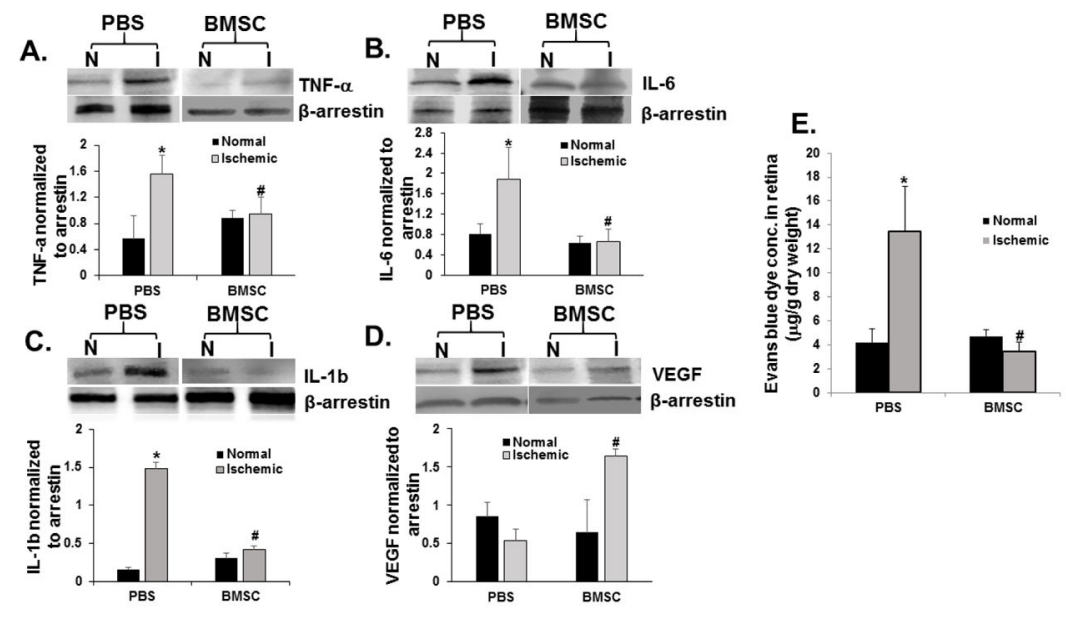

\begin{abstract}
Figure 6.
Evaluation of inflammatory and vascular permeability markers. Representative Western blot illustrating TNFa (A), IL6 (B), IL1 $\beta$ (C), and VEGF (D). BMSC treatment significantly reduced levels of TNFa, IL1 $\beta$, and IL6; and increased VEGF compared to PBS injected ischemic eyes. The bar graphs demonstrate the levels of all four proteins normalized to $\beta$ arrestin. (E) Bar graph exhibiting Evans Blue dye extravasation, marker of vascular permeability, in ischemic PBS and BMSC injected retina. Data are shown as mean \pm SEM. * indicates $\mathrm{P}<0.05$ compared to the paired normal retina within a group (PBS or BMSC); \# indicates $\mathrm{P}<0.05$ between the ischemic retinae of PBS and BMSC. $\mathrm{N}=3-4$ per group for Western blotting, and 7 for Evans Blue. See Figure 4 legend for abbreviations.
\end{abstract}




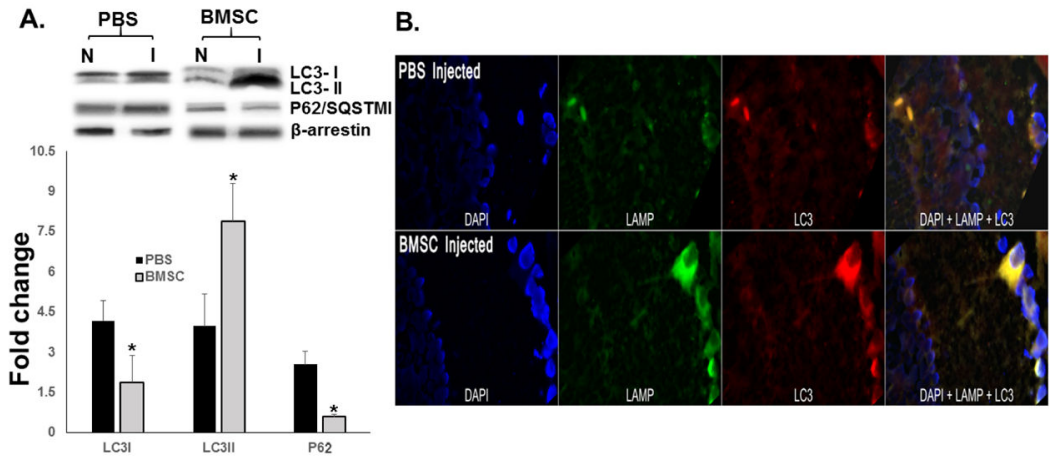

Figure 7.

(A) Western blotting for LC3-I, LC3-II, and p62 as markers of autophagy. The results are expressed as fold change from normal to ischemic within each group, with protein levels normalized to $\beta$-arrestin. $\mathrm{N}=5$ per group. Data are shown as mean $\pm \mathrm{SEM}$. There were significant decreases in LC3-I and in p62, and significant increases in LC3-II in the BMSC group. * indicates $\mathrm{P}<0.05$ compared to the paired normal retina within a group (PBS or BMSC); \# indicates $\mathrm{P}<0.05$ between the ischemic retinae of PBS and BMSC. (B) Retinal cryosections stained for DAPI (blue), LAMP (red), and antibody specific for LC3-II (green), in PBS injected (top), and BMSC injected (bottom) ischemic retinae. The images demonstrate that LC3-II and LAMP co-localize in cytoplasm, indicating their localization into autophagosomes, confirming the presence of autophagy. See Methods for more details. 